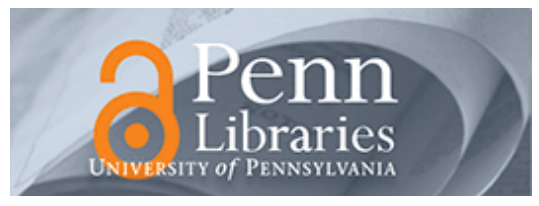

University of Pennsylvania

ScholarlyCommons

3-1-1990

\title{
Resistance Distributions of the Random Resistor Network Near the Percolation Threshold
}

\author{
A. Brooks Harris \\ University of Pennsylvania, harris@sas.upenn.edu \\ Yigal Meir
}

Amnon Aharony

Follow this and additional works at: https://repository.upenn.edu/physics_papers

Part of the Physics Commons

\section{Recommended Citation}

Harris, A., Meir, Y., \& Aharony, A. (1990). Resistance Distributions of the Random Resistor Network Near the Percolation Threshold. Physical Review B, 41 (7), 4610-4618. http://dx.doi.org/10.1103/

PhysRevB.41.4610

At the time of publication, author A. Brooks Harris was affiliated with Tel Aviv University. Currently, he is a faculty member in the Physics Department at the University of Pennsylvania.

This paper is posted at ScholarlyCommons. https://repository.upenn.edu/physics_papers/371

For more information, please contact repository@pobox.upenn.edu. 


\title{
Resistance Distributions of the Random Resistor Network Near the Percolation Threshold
}

\begin{abstract}
We study the generalized resistive susceptibility, $\chi(\lambda) \equiv \Sigma_{x^{\prime}}\left[\exp \left[-1 / 2 \lambda^{2} R\left(x x^{\prime}\right)\right]\right]_{a v}$ where []$_{a v}$ denotes an average over all configurations of clusters with weight appropriate to bond percolation, $R\left(\mathrm{x}, \mathrm{x}^{\prime}\right)$ is the resistance between nodes $\mathrm{x}$ and $\mathrm{x}^{\prime}$ when occupied bonds are assigned unit resistance and vacant bonds infinite resistance. For bond concentration $p$ near the percolation threshold at $p_{c}$, we give a simple calculation in $6-\varepsilon$ dimensions of $\chi(\lambda)$ from which we obtain the distribution of resistances between two randomly chosen terminals. From $\chi(\lambda)$ we also obtain the qth-order resistive susceptibility $\chi^{(q)} \equiv \Sigma_{x^{\prime}}\left[\nu\left(x, x^{\prime}\right)\right.$ $\left.R\left(\mathrm{x}, \mathrm{x}^{\prime}\right)^{\mathrm{q}}\right]_{\mathrm{av}}$, where $v\left(\mathrm{x}, \mathrm{x}^{\prime}\right)$ is an indicator function which is unity when sites $\mathrm{x}$ and $\mathrm{x}^{\prime}$ are connected and is zero otherwise. In the latter case, $v\left(\mathrm{x}, \mathrm{x}^{\prime}\right) R\left(\mathrm{x}, \mathrm{x}^{\prime}\right)^{\mathrm{q}}$ is interpreted to be zero. Our universal amplitude ratios, $\rho_{\mathrm{q}} \equiv \lim _{\mathrm{p} \rightarrow \mathrm{pc}} \chi^{(\mathrm{q})}\left(\chi^{(0)}\right)^{\mathrm{q}-1}\left(\chi^{(1)}\right)^{\mathrm{q}}$, reproduce previous results and agree beautifully with our new lowconcentration series results. We give a simple numerical approximation for the $\chi^{(q)}$ 's in all dimensions. The relation of the scaling function for $\chi(\lambda)$ with that for the susceptibility of the diluted $x y$ model for $p$ near $p_{\mathrm{c}}$ is discussed.

Disciplines

Physics

\section{Comments}

At the time of publication, author A. Brooks Harris was affiliated with Tel Aviv University. Currently, he is a faculty member in the Physics Department at the University of Pennsylvania.
\end{abstract}




\title{
Resistance distributions of the random resistor network near the percolation threshold
}

\author{
A. B. Harris, ${ }^{*}$ Yigal Meir, and Amnon Aharony \\ Raymond and Beverly Sackler Faculty of Exact Sciences, School of Physics and Astronomy, Tel Aviv University, \\ Ramat Aviv, Tel Aviv 69978, Israel
}

(Received 8 June 1988; revised manuscript received 11 July 1989)

\begin{abstract}
We study the generalized resistive susceptibility, $\chi(\lambda) \equiv \Sigma_{\mathrm{x}} \cdot\left[\exp \left[-\frac{1}{2} \lambda^{2} R\left(\mathbf{x x}^{\prime}\right)\right]\right]_{\mathrm{av}}$ where []$_{\mathrm{av}}$ denotes an average over all configurations of clusters with weight appropriate to bond percolation, $R\left(\mathbf{x}, \mathbf{x}^{\prime}\right)$ is the resistance between nodes $\mathbf{x}$ and $\mathbf{x}^{\prime}$ when occupied bonds are assigned unit resistance and vacant bonds infinite resistance. For bond concentration $p$ near the percolation threshold at $p_{c}$, we give a simple calculation in 6- $\epsilon$ dimensions of $\chi(\lambda)$ from which we obtain the distribution of resistances between two randomly chosen terminals. From $\chi(\lambda)$ we also obtain the $q$ th-order resistive susceptibility $\chi^{(q)} \equiv \Sigma_{\mathbf{x}}\left[v\left(\mathbf{x}, \mathbf{x}^{\prime}\right) R\left(\mathbf{x}, \mathbf{x}^{\prime}\right)^{q}\right]_{\text {av }}$, where $v\left(\mathbf{x}, \mathbf{x}^{\prime}\right)$ is an indicator function which is unity when sites $x$ and $x^{\prime}$ are connected and is zero otherwise. In the latter case, $v\left(x, x^{\prime}\right) R\left(x, x^{\prime}\right)^{q}$ is interpreted to be zero. Our universal amplitude ratios, $\rho_{q} \equiv \lim _{p \rightarrow p_{c}} \chi^{(q)}\left(\chi^{(0)}\right)^{q-1}\left(\chi^{(1)}\right)^{q}$, reproduce previous results and agree beautifully with our new low-concentration series results. We give a simple numerical approximation for the $\chi^{(q)}$ 's in all dimensions. The relation of the scaling function for $\chi(\lambda)$ with that for the susceptibility of the diluted $x y$ model for $p$ near $p_{c}$ is discussed.
\end{abstract}

\section{INTRODUCTION}

The geometrical properties of clusters formed by randomly diluting a lattice are by now well established. ${ }^{1-3}$ In the percolation model which we study, each bond is independently occupied with probability $p$ and vacant with probability $1-p$. Likewise, the randomly diluted resistor network (RRN) in which occupied bonds have conductance $\sigma$ and vacant bonds conductance 0 has been widely studied for more than a decade. ${ }^{4-10}$ In spite of this, relatively little is known about the exact form of the probability distribution $P_{c}(R, \mathrm{x})$ for the resistance $R$ between two nodes known to be in the same cluster at separation $x$. Note that this is a conditional probability, conditioned by the restriction that the two sites be in the same cluster. Formally, this quantity is defined as

$$
P_{c}(R, \mathbf{x})=P(R, \mathbf{x}) /[v(0, \mathbf{x})]_{\mathrm{av}},
$$

where $[\cdots]_{\mathrm{av}}$ denotes an average over all configurations of occupied and unoccupied bonds, $v\left(\mathbf{x}, \mathbf{x}^{\prime}\right)$ is an indicator function which is unity if sites $x$ and $x^{\prime}$ are in the same cluster and is zero otherwise, and $P(R, \mathbf{x})$ is the bare (i.e., unconditional) probability that $R(0, \mathbf{x})$ assume the value $R$ :

$$
P(R, \mathrm{x})=[\delta(R(0, \mathrm{x})-R) v(0, \mathrm{x})]_{\mathrm{av}} .
$$

In early work, ${ }^{11}$ it was realized that under transformation of length scales there are three regimes, $p<p_{c}$, $p=p_{c}$, and $p>p_{c}$, where $p_{c}$ is the percolation threshold. For $p<p_{c}$ the conductance $\sigma$ scales to zero, whereas for $p>p_{c} \sigma$ scales to a constant. In these cases $P(R, \mathbf{x})$ on large length scales becomes a $\delta$ function, as expected for a pure system with fixed $\sigma$. For $p=p_{c}$, however, $P(R, \mathbf{x})$ becomes a distribution invariant to change of length scale. The form of $P(R, \mathrm{x})$ at $p=p_{c}$ was obtained numer- ically in $d=2$ spatial dimensions by a real-space renormalization group. ${ }^{11}$ Also, the first three moments of the conductivity were studied as a function of length scale by Rammal et al. ${ }^{12}$ However, the exact relation between their distribution of conductances and the distribution $P(R, \mathrm{x})$ is not obvious. Finally, within mean-field theory (i.e., for $d>6$ ) one has ${ }^{13}$ for $p=p_{c}$

$$
\begin{aligned}
P_{c}(R, \mathbf{x})= & \frac{1}{R} \frac{1}{[(d-4) / 2] !}\left(\frac{b x^{2}}{4 \sigma R}\right)^{(d-2) / 2} \\
& \times \exp \left[-b x^{2} /(4 \sigma R)\right],
\end{aligned}
$$

where $b$ is a constant of order unity.

With this paper we initiate a program to obtain information on $P(R, x)$ within (a) the renormalization-group $\epsilon$ expansion and (b) low-concentration series. We present a formulation from which $P_{c}(R, \mathbf{x})$ and $P(R, \mathbf{x})$ can be determined. The behavior of these quantities as functions of $R$ and $x$ is quite complex and is analyzed elsewhere. ${ }^{14,15}$ Here we concentrate on simpler quantities which result when $x$ is summed over. For instance, a quantity we consider is $P_{c}(R)$, the distribution of resistances between randomly chosen pairs of points on a percolation cluster.

There are two plausible ways one might define such a normalized distribution function. The first definition can be described in terms of the following numerical simulation. One randomly occupies bonds in a very large system. Thereby, the system will contain an ensemble of clusters, which, if the system is large enough, will reproduce the true distribution of clusters. Now construct a histogram which shows the number of occurrences of resistances in intervals, say, between $R$ and $R+d R$, over the complete ensemble of pairs of nodes $\mathbf{x}$ and $\mathbf{x}^{\prime}$ conditional on these points being in the same cluster. The histogram, when normalized by dividing by the total num- 
ber of occurrences, will be defined to be $P_{c}^{\mathrm{I}}(R)$. In the second definition the average is performed in a slightly different way. Here one considers a given site $i$ and constructs the normalized distribution function $P_{i}(R)$ for resistances between site $i$ and all other sites in the same cluster. Since this distribution depends on the choice of site $i$, it is natural to average over possible choices, whence we define $P_{c}^{\mathrm{II}}(R)=\left[P_{i}(R)\right]_{\mathrm{av}}$.

It is not immediately obvious which of these definitions is the more useful. It is therefore helpful to express them in terms of averages over clusters. In case $I$ it is seen that

$$
P_{c}^{\mathrm{I}}(R)=\left(\sum_{\mathbf{x}, \mathbf{x}^{\prime}} v\left(\mathbf{x}, \mathbf{x}^{\prime}\right) \delta\left(R-R\left(\mathbf{x}, \mathbf{x}^{\prime}\right)\right)\right]_{\mathrm{av}} /\left[\sum_{\mathbf{x}, \mathbf{x}^{\prime}} v\left(\mathbf{x}, \mathbf{x}^{\prime}\right)\right]_{\mathrm{av}} .
$$

For $p<p_{c}$ one can express the averages as sums over all possible clusters $\Gamma$, each occurring with weight $P(\Gamma) \equiv p^{s(\Gamma)}(1-p)^{t(\Gamma)}$, where $s(\Gamma)$ is the number of occupied bonds in $\Gamma$ and $t(\Gamma)$ is the number of vacant perimeter bonds adjacent to $\Gamma$. We have

$$
P_{c}^{\mathrm{I}}(R)=\sum_{\Gamma}\left[P(\Gamma) \sum_{\mathbf{x}, \mathbf{x}^{\prime} \in \Gamma} \delta\left(R-R\left(\mathbf{x}, \mathbf{x}^{\prime}\right)\right)\right] / \sum_{\Gamma} P(\Gamma) s(\Gamma)^{2}
$$

The natural extension of this formula for $p>p_{c}$ is

$$
P_{c}^{\mathrm{I}}(R)=P_{c}^{\infty}(R)
$$

where $P_{c}^{\infty}(R)$ is the normalized distribution for $R\left(\mathbf{x}, \mathbf{x}^{\prime}\right)$ when $x$ and $x^{\prime}$ are on the same cluster but are infinitely far apart. Similarly, in case II we write

$P_{c}^{\mathrm{II}}(R)=\left(\sum_{\mathbf{x}^{\prime}} v\left(\mathbf{x}, \mathbf{x}^{\prime}\right) \delta\left(R-R\left(\mathbf{x}, \mathbf{x}^{\prime}\right)\right) / \sum_{\mathbf{x}^{\prime}} v\left(\mathbf{x}, \mathbf{x}^{\prime}\right)\right)_{\mathrm{av}}$.

In terms of cluster sums this result is

$$
\begin{aligned}
P_{c}^{\mathrm{II}}(R)= & P_{\infty} P_{c}^{\infty}(R) \\
& +\sum_{\Gamma}\left(P(\Gamma) \sum_{\mathbf{x}, \mathbf{x}^{\prime} \in \Gamma} \delta\left(R-R\left(\mathbf{x}, \mathbf{x}^{\prime}\right)\right) / s(\Gamma)\right),
\end{aligned}
$$

where $P_{\infty}$ is the probability that a site is not in a finitesized cluster. Comparing the expressions for $P_{c}^{\mathrm{I}}(R)$ and $P_{c}^{\text {II }}(R)$ we see that the former involves an average with an additional power of $s(\Gamma)$ and thereby weights more heavily larger clusters of sites. Thus, $P_{c}^{\mathrm{I}}(R)$ provides a better characterization of large clusters. Also, the averages required to calculate this quantity are simpler than those for $P_{c}^{\mathrm{II}}(R)$. Accordingly, we restrict our attention to $P_{c}^{\mathrm{I}}(R)$, which we henceforth denote simply $P_{c}(R)$ :

$$
P_{c}(R)=\sum_{\mathbf{x}}[v(0, \mathbf{x}) \delta(R-R(0, \mathbf{x}))]_{\mathrm{av}} / \chi_{p},
$$

where $\chi_{p}=\Sigma_{x}[v(0, \mathbf{x})]_{\mathrm{av}}$ is the percolation susceptibility.

Up to now $P_{c}(R)$ has only been studied through its moments, $\chi^{(q)}$ defined as

$$
\begin{aligned}
\chi^{(q)} & \equiv \sum_{\mathbf{x}}\left[v\left(\mathbf{x}, \mathbf{x}^{\prime}\right) R\left(\mathbf{x}, \mathbf{x}^{\prime}\right)^{q}\right]_{\mathrm{av}} \\
& =\chi_{p} \int_{0}^{\infty} P_{c}(R) R^{q} d R,
\end{aligned}
$$

where $v R^{q}$ is taken to be zero when $v$ vanishes. $\chi^{(q)}$ is referred to as the $q$ th moment resistive susceptibility and is known to diverge for $p \rightarrow p_{c}$ as

$$
\chi^{(q)} \sim\left|p_{c}-p\right|^{-\gamma-q \phi},
$$

where $\gamma$ is the susceptibility exponent of percolation ${ }^{16}$ and $\phi$ is the resistance crossover exponent. ${ }^{9,10}$ Furthermore there are calculations ${ }^{13}$ within the renormalizationgroup $\epsilon$ expansion for the universal amplitude ratios ${ }^{17}$

$$
\rho_{q} \equiv \lim _{p \rightarrow p_{c}} \chi^{(q)}\left[\chi^{(0)}\right]^{q-1} /\left[\chi^{(1)}\right]^{q},
$$

where $\chi^{(0)}=\chi_{p}$. Here we give the first numerical calculations of $\chi^{(q)}$ in high dimensions. We determine several of the $\rho_{q}$ 's from low-concentration series and find excellent agreement with the $\epsilon$ expansion. From these numerical results we formulate a simple numerical approximation for the $\rho$ 's that is quite accurate in all dimensions.

\section{CALCULATION OF THE GENERALIZED RESISTIVE SUSCEPTIBILITY AND DERIVED QUANTITIES}

A. Formulation for $d=6-\epsilon$

Here we give calculations of the generalized resistive susceptibility

$$
\begin{aligned}
\chi_{R}(\lambda) & \equiv \sum_{\mathbf{x}} G\left(\mathbf{x}, \mathbf{x}^{\prime} ; \lambda\right) \\
& \equiv \sum_{\mathbf{x}}\left[\exp \left[-\frac{1}{2} \lambda^{2} R\left(\mathbf{x}, \mathbf{x}^{\prime}\right)\right]\right]_{\mathrm{av}}
\end{aligned}
$$

using the $\epsilon$-expansion, where $\epsilon=6-d . \chi^{(q)}$ can be obtained from the coefficient of $\lambda^{2 q}$ in the Taylor-series expansion of $\chi_{R}(\lambda)$ as will be shown in Eq. (38a). We use the field theory proposed by Stephen ${ }^{18}$ in the version discussed in detail by Harris and Lubensky (HL). ${ }^{13}$ In this formulation the field-theoretic free energy is of the form

$$
\begin{aligned}
& F= \frac{1}{2} \sum_{\mathbf{q}} \sum_{\lambda}^{\prime} \Psi_{\lambda}(\mathbf{q}) \Psi_{-\lambda}(-q)\left[r(\lambda)+q^{2}\right] \\
&+\frac{1}{6} u_{3} \sum_{\mathbf{q}_{1}, \mathbf{q}_{2}{ }^{\prime} \lambda_{1}, \lambda_{2}} \sum_{\lambda_{1}}\left(\mathbf{q}_{1}\right) \Psi_{\lambda_{2}}\left(\mathbf{q}_{2}\right) \\
& \\
& \quad \times \Psi_{-\lambda_{1}-\lambda_{2}}\left(-\mathbf{q}_{1}-\mathbf{q}_{2}\right),
\end{aligned}
$$

where $\Psi_{\lambda}(\mathrm{q})$ are Gaussian variables with $\mathrm{q}$ restricted to 
lie within the continuum of a unit sphere and $\lambda$ represents an $n$-component vector, $\left(\lambda_{1}, \lambda_{2}, \ldots, \lambda_{n}\right)$, each component of which is allowed to assume values on a discrete mesh of points over a finite range, $-\lambda_{\max }<\lambda_{\alpha}<\lambda_{\max }$. For the RRN the appropriate limit to consider is that in which $\lambda_{\max }$ becomes infinite and the mesh size goes to zero. Thus for the RRN sums over $\lambda$ can be replaced by integrals in which each of the $n$ components of $\lambda$ ranges from $-\infty$ to $+\infty$. The interpretation of $\Psi_{\lambda}(q)$ is that it describes the fluctuation at wave vector $\mathbf{q}$ of the quantity $\Pi_{j} \exp \left(i \lambda_{j} V_{j}\right)$, where the product is over all replicas $j$, so that

$$
\left\langle\Psi_{\lambda}(\mathbf{x}) \Psi_{-\lambda}\left(\mathbf{x}^{\prime}\right)\right\rangle=G\left(\mathbf{x}, \mathbf{x}^{\prime} ; \lambda\right) .
$$

Since the variable $\Psi_{\lambda}(q)$ is trivially unity when $\lambda=0$, this value of $\lambda$ is not allowed, and in Eq. (14) the primes indicate that terms for which any $\lambda=0$ is excluded from the sums. Also in Eq. (14)

$$
r(\lambda)=r_{0}+w_{1} \lambda^{2}+w_{2} \lambda^{4} \cdots,
$$

where $r_{0}=a_{0}\left(p_{0}-p\right)$, where $p_{0}$ is the mean-field value of the percolation threshold, $a_{0}$ is a constant of order unity, and $w_{1}=1 / \sigma$ for the RRN and $w_{1}=T / J$ for the randomly diluted $x y$ (RXY) model at low temperature $T$ near the percolation threshold, where $J$ is the exchange constant. Below we will often indicate dependence on $p$, sometimes representing it by $r_{0}$ or by $t=a_{0}\left(p_{c}-p\right)$. As discussed in some detail in HL, the percolation problem is recovered for $\lambda=0$, so that we have

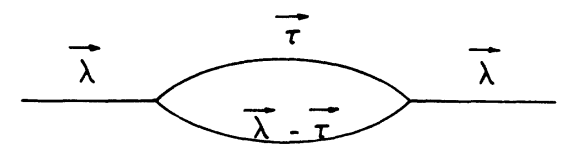

FIG. 1. Diagram that contributes to the susceptibility of the one-loop order.

$$
\lim _{\lambda \rightarrow 0} G\left(\mathbf{x}, \mathbf{x}^{\prime} ; \lambda\right)=G_{p}\left(\mathbf{x}, \mathbf{x}^{\prime}\right) \equiv\left[v\left(\mathbf{x}, \mathbf{x}^{\prime}\right)\right]_{\mathrm{av}},
$$

where $G_{p}\left(\mathbf{x}, \mathbf{x}^{\prime}\right)$ is the percolation correlation function, i.e., the probability that the points $x$ and $x^{\prime}$ are in the same cluster.

We now carry out a one-loop calculation ${ }^{19}$ involving the diagram shown in Fig. 1 for $d=6-\epsilon$. For this purpose, since we do not keep track of corrections to scaling, we set $u_{3}$ equal to the correct value for a diagrammatic evaluation at the percolation threshold. To first order in $\epsilon$, this value is the same as the fixed point value of $u_{3}$ in the momentum-shell recursion relations, ${ }^{16}$ i.e.,

$$
u_{3}^{2}=2 \epsilon /\left(7 K_{d}\right) \text {, }
$$

where $K_{d}$ is the phase-space factor in $d$ dimensions, whose exact value is not needed here. We could give an argument to indicate that the $w_{k}$ 's with $k \geq 1$ are irrelevant, but this was shown by HL, so we simplify the discussion by assuming this fact here. Then from the diagram of Fig. 1 we have that the spatial Fourier transform of $G\left(\mathbf{x}, \mathbf{x}^{\prime} ; \lambda\right)$ is

$$
\hat{G}^{-1}\left(r_{0}, \mathbf{q}, \lambda\right)=r_{0}+q^{2}+w_{1} \lambda^{2}-\frac{1}{2} u_{3}^{2} \int_{0}^{1} d \mathbf{k}\left(\sum_{\tau}^{\prime} \frac{1}{\left[r_{0}+\left(\mathbf{k}+\frac{1}{2} \mathbf{q}\right)^{2}+w_{1} \tau^{2}\right]\left[r_{0}+\left(\mathbf{k}-\frac{1}{2} \mathbf{q}\right)^{2}+w_{1}(\tau-\lambda)^{2}\right]}\right),
$$

where the prime on the summation indicates the terms for which either $\tau=\lambda$ or $\tau=0$ are omitted. If we apply Eq. (18) at the critical point $(\mathbf{q}=\lambda=0)$, where $\hat{G}^{-1}=\chi_{p}^{-1}=0$, then we identify the critical value of $r_{0}$, i.e., $r_{0 c}$. Subtracting this equation from that written in Eq. (18) we obtain

$$
\begin{aligned}
\hat{G}^{-1}\left(r_{0}, \mathbf{q}, \lambda\right)=t+q^{2}+w-\frac{1}{2} u_{3}^{2} \int_{0}^{1} d \mathbf{k} & \frac{1}{k^{4}}-\frac{2}{\left[t+\left(\mathbf{k}+\frac{1}{2} \mathbf{q}\right)^{2}+w\right]\left[t+\left(\mathbf{k}-\frac{1}{2} \mathbf{q}\right)^{2}\right]} \\
& \left.+\sum_{\tau} \frac{1}{\left[t+\left(\mathbf{k}+\frac{1}{2} \mathbf{q}\right)^{2}+w_{1} \tau^{2}\right]\left[t+\left(\mathbf{k}-\frac{1}{2} \mathbf{q}\right)^{2}+w_{1}(\tau-\lambda)^{2}\right]}\right),
\end{aligned}
$$

where $t=r_{0}-r_{0 c}=a_{0}\left(p_{c}-p\right)$ and we introduced the shorthand notation $w$ to denote $w_{1} \lambda^{2}$. Since $r_{0 c}$ is of order $u_{3}^{2}$, replacing $r_{0}$ by $t$ in the integrals affects only terms of higher order in $\epsilon$. Also, to obtain Eq. (19), we used $\sum_{\tau}\left(k^{2}+w_{1} \tau^{2}\right)^{-2}=k^{-4}$, for $n \rightarrow 0$ [c.f. Eq. (21b), following]. In separate papers the analyses based on Eq. (19) of (a) $\hat{G}(t=0, \mathbf{q}, \lambda)$ and a resulting evaluation ${ }^{14}$ of $P(R, \mathbf{x})$ and (b) the phase diagrams of the RRN and RXY models ${ }^{15}$ for $p$ near $p=p_{c}$ are presented.

\section{B. Scaling form for the resistive susceptibility $\chi(\lambda)$}

Here we develop the scaling form for $\hat{G}\left(r_{0}, q=0 ; \lambda\right)=\chi_{R}(\lambda)$, which we now denote $\chi_{R}(t, \lambda)$ to emphasize the dependence on $p$ through the variable $t$. To do this we set $q=0$ in Eq. (19). Our results are consistent with those of HL who used a more complicated method. The principal difficulty in evaluating the right-hand side of Eq. (19) involves the sums over $\tau$. Following HL we write

$$
S \equiv \sum_{\tau_{1}, \tau_{2}, \ldots, \tau_{n}}\left[D+w_{1} \tau^{2}\right]^{-1}\left[D+w_{1}(\tau-\lambda)^{2}\right]^{-1}
$$


where $D=t+k^{2}$. We have

$$
S=\sum_{\tau_{1}, \tau_{2}, \ldots, \tau_{n}} \int_{0}^{\infty} \exp \left[-D \mu-\mu w_{1} \tau^{2}\right] d \mu \int_{0}^{\infty} \exp \left[-D v-v w_{1}(\tau-\lambda)^{2}\right] d v
$$

and replacing the sums by integrals, we have, for $n \rightarrow 0$,

$$
S=\frac{1}{2} \int_{-1}^{1} d \rho\left[D+\frac{1}{4} w\left(1-\rho^{2}\right)\right]^{-2} .
$$

For $w \equiv w_{1} \lambda^{2}=0$, we see that $S=\left(t+k^{2}\right)^{-2}$, as used above. Now for $\mathbf{q}=0$ the integral over momentum $\mathbf{k}$ in Eq. (19) can be expressed as

$$
\chi_{R}^{-1}(t, \lambda)=t+w-\frac{1}{8} K_{d} u_{3}^{2} \int_{0}^{1} x^{2} d x \int_{-1}^{1} d \rho\left(\frac{1}{x^{2}}-\frac{2}{(x+t)\left(x+b_{1}\right)}+\frac{1}{\left(x+b_{2}\right)^{2}}\right)
$$

with $b_{1}=t+w$ and $b_{2}=t+w\left(1-\rho^{2}\right) / 4$. These integrations are easily performed. In the result we drop terms which are second order in the small quantities $t$ and $w$ and set $K_{d} u_{3}^{2}=2 \epsilon / 7$. Thereby we obtain the scaling form for $\chi^{-1}$ :

$$
\begin{aligned}
\chi_{R}^{-1}(t, \lambda)= & t+w+\frac{\epsilon}{7} t \ln t+\frac{5 \epsilon}{42} w \ln t \\
& +\frac{\epsilon}{7} t\left(\frac{(1+x)^{2}}{x} \ln (1+x)-\frac{1}{2}-\frac{x}{12}-\frac{1}{2} \int_{-1}^{1} d \rho\left[1+\frac{1}{4} x\left(1-\rho^{2}\right)\right] \ln \left[1+\frac{1}{4} x\left(1-\rho^{2}\right)\right]\right),
\end{aligned}
$$

where $x=w / t$. We now exponentiate the results so that $t[1+(\epsilon / 7) \ln t] \rightarrow t^{\gamma}$ and $w[1+(5 \epsilon / 42) \ln t] \rightarrow w t^{\gamma-\phi}$, where $\gamma=1+\epsilon / 7$ and $\phi=1+\epsilon / 42$ are the known values of the susceptibility exponent for percolation ${ }^{16}$ and the resistance crossover exponent, ${ }^{9,10}$ respectively. We fix the constants in this scaling function by requiring the function $F(y)$ in

$$
\chi_{R}^{-1}(t, \lambda)=A t^{\gamma} F(y)
$$

to obey $F(0)=F^{\prime}(0)=1$. Thus with $A=1+\epsilon / 14$ and $y=(1+3 \epsilon / 28) w / t^{\phi}$ we have

$$
\begin{aligned}
F(y) & =1+y+\frac{\epsilon}{7}\left[\frac{(1+y)^{2}}{y} \ln (1+y)-1-\frac{4 y}{3}-\frac{1}{2} \int_{-1}^{1} d \rho\left(1+\frac{y}{4}\left(1-\rho^{2}\right)\right] \ln \left(1+\frac{y}{4}\left(1-\rho^{2}\right)\right)\right] \\
& \equiv 1+y+\frac{\epsilon}{7} H(y),
\end{aligned}
$$

where

$$
H(y)=\frac{(1+y)^{2}}{y} \ln (1+y)+\frac{1}{3}-\frac{19 y}{18}-\left(\frac{(y+4)^{3 / 2}}{3 y^{1 / 2}}\right) \ln \left(\frac{\sqrt{4+y}+\sqrt{y}}{2}\right) .
$$

Both here and in the HL calculation one uses general scaling arguments to show that $F$ is a function of the scaling variable $y=a w / t^{\phi}=a w_{1} \lambda^{2} / t^{\phi}=a \lambda^{2} /\left(\sigma t^{\phi}\right)$. To order $\epsilon$, one cannot distinguish in $H(y)$ between $w / t$ and $w / t^{\phi}$. Also, no conclusions should be drawn concerning the values of $A$ and $a$. These are nonuniversal constants whose values depend on the details of the interactions and the exact cutoff introduced in the calculations. A more general calculation of $\hat{G}^{-1}$ for nonzero wave vector $\mathbf{q}$, from which information on the distribution of $R\left(\mathbf{x}, \mathbf{x}^{\prime}\right)$ for fixed $\mathbf{x}-\mathbf{x}^{\prime}$ can be obtained, will be reported elsewhere. ${ }^{14}$ Here we get only $\mathbf{q}=0$ information. We can compare this simplified calculation with that of $\mathrm{HL}$ as follows. They give

$$
\chi_{R}^{-1}(t, \lambda)=\Gamma_{0}+\Gamma_{1} \lambda^{2}+\Gamma_{2} \lambda^{4}+\cdots,
$$

where the leading behavior of $\Gamma_{k}$ is given by $\Gamma_{k}=\delta_{k} A b^{k} t^{\gamma-k \phi}$. This result is the most general one consistent with their results for the universal amplitude ratios $\delta_{k}$, which were given as

$$
\delta_{k} \equiv \frac{\Gamma_{k}\left[\Gamma_{0}\right]^{k-1}}{\Gamma_{1}^{k}}=\frac{\epsilon}{7}(-1)^{k}\left(\frac{2}{k\left(k^{2}-1\right)}-\frac{1}{k\left(k^{2}-1\right)} \frac{k !(k+1) !}{(2 k+1) !}\right), \quad k \geq 2,
$$

and we adopt the convention that $\delta_{0}=\delta_{1}=1$. We therefore identify $y$ as $y=b \lambda^{2} / t^{\phi}$. Then Eq. (27) becomes

$$
\chi_{R}^{-1}(t, \lambda)=A t^{\gamma}\left[1+y+\frac{\epsilon}{7} \sum_{k=2}^{\infty}(-y)^{k}\left[\frac{2}{k\left(k^{2}-1\right)}-\frac{1}{k\left(k^{2}-1\right)} \frac{k !(k+1) !}{(2 k+1) !}\right]\right] .
$$


It can be verified that the function in the large parentheses of Eq. (29) is the power-series expansion of $F(y)$ in Eq. (25). The final result, therefore, is that

$$
\begin{aligned}
\chi_{R}(t, \lambda) & =\sum_{\mathbf{x}}\left[\exp \left[-\frac{1}{2} \lambda^{2} R(0, \mathbf{x})\right]\right]_{\mathrm{av}} \\
& =A^{-1} t^{-\gamma}\left[1+y+\frac{\epsilon}{7} H(y)\right]^{-1},
\end{aligned}
$$

where again $y=a \lambda^{2} /\left(\sigma t^{\phi}\right)$. This is a universal result in that models with different short-ranged interactions (e.g., with nonzero conductances $\sigma_{2}$ between next-nearest neighbors) will still be described by Eq. (30) although different values of the scale factors, $A$ and $a$, will be required.

\section{Resistance distribution for $p$ near $p_{c}$}

We now see what information is contained in $\chi_{R}(t, \lambda)$. As pointed out by HL the universal amplitude ratios $\rho_{q}$ defined in Eq. (12) are interesting quantities. In Sec. III we will compare the $\epsilon$-expansion results for their values with those we find from the low-concentration series. Here we focus on the distribution for resistances between all pairs of sites, $P_{c}(R)$, defined in Eq. (9). To study this quantity we write

$$
\begin{aligned}
\chi_{R}(t, \lambda) & =\sum_{\mathbf{x}}\left[\exp \left[-\frac{1}{2} \lambda^{2} R(0, \mathbf{x})\right]\right]_{\mathrm{av}} \\
& =\sum_{\mathbf{x}} \int_{0}^{\infty} \exp \left(-\frac{1}{2} \lambda^{2} R\right) P(R, \mathbf{x}) d R .
\end{aligned}
$$

Taking the inverse Laplace transform of this relation leads to

$$
\begin{aligned}
\frac{1}{2 \pi i} \int_{b-i \infty}^{b+i \infty} \chi_{R}(t, \lambda) \exp \left(\frac{1}{2} \lambda^{2} R\right) d\left(\frac{1}{2} \lambda^{2}\right) \\
=\sum_{\mathbf{x}}[\delta(R-R(0, \mathbf{x}))]_{\mathrm{av}}=P_{c}(R) \chi_{p},
\end{aligned}
$$

where $b$ is real and positive. Thus, $P_{c}(R)$ is essentially the inverse Laplace transform of $\chi_{R}(t, \lambda)$. Using Eq. (30) and with $z=a \lambda^{2} /\left(\sigma t^{\phi}\right)$ we write Eq. (32) as

$$
P_{c}(R)=\frac{1}{2 \pi i} \int_{b-i \infty}^{b+i \infty} \frac{\exp \left[z \sigma R t^{\phi} /(2 a)\right] \sigma t^{\phi}}{[1+z+(\epsilon / 7) H(z)] 2 a} d z
$$

in the notation of Eq. (26). Thus $P_{c}(R)$ can be written in the form

$$
P_{c}(R)=\frac{\sigma t^{\phi}}{2 a} \Lambda\left(\frac{\sigma R t^{\phi}}{2 a}\right) \equiv \frac{1}{R^{*}(t)} \Lambda\left(\frac{R}{R^{*}(t)}\right),
$$

where $R^{*}(t)=2 a /\left(\sigma t^{\phi}\right)$ and

$$
\Lambda(x)=\frac{1}{2 \pi i} \int_{b-i \infty}^{b+i \infty} \frac{e^{z x}}{[1+z+(\epsilon / 7) H(z)]} d z .
$$

Note that $P_{c}(R)$ has only one scale factor, $a$, because the amplitude is fixed by the condition $\int_{0}^{\infty} P_{c}(R) d R=1$.

To evaluate Eq. (34) we deform the contour of integration to surround the cut along the negative real $z$ axis, as shown in Fig. 2 and as discussed in the Appendix. We

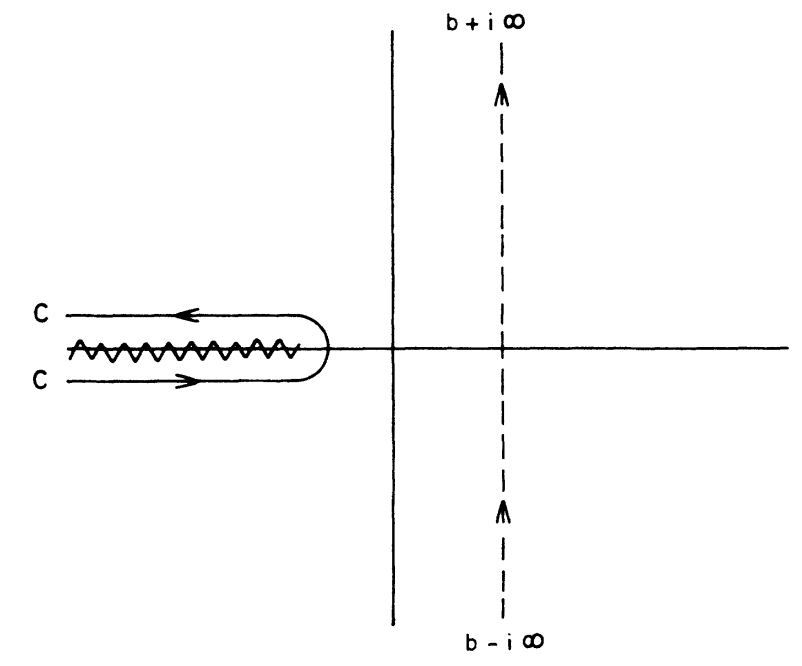

FIG. 2. Original contour of integration (dashed line) in Eq. (35). The deformed contour is shown by a solid line surrounding the branch cut (wavy line) from $z=-1$ to $-\infty$.

thereby find two contributions: one from the region near $z=-1$ where for $\epsilon=0$ there was a simple pole, and the other from the remaining part of the branch cut. We refer to these two contributions as the "pole" and "cut" contributions, respectively, and write

$$
P_{c}(R)=P_{c}(R)_{\text {pole }}+P_{c}(R)_{\text {cut }} \text {. }
$$

The pole contribution is found from the solution of

$$
\operatorname{Re}[1+z+(\epsilon / 7) H(z)]=0
$$

to order $\epsilon$. In this way one finds the pole to be shifted to

$$
z_{0} \sim-1-(\epsilon / 7) \operatorname{Re} H(-1) .
$$

For $z$ near $z_{0}$ the denominator in Eq. (35) may be represented as

$$
\left(z-z_{0}\right)[1+(\epsilon / 7)(d \operatorname{Re} H(-1) / d z)] .
$$

The pole contribution to $P_{c}(R)$ is

$$
\begin{aligned}
{\left[P_{c}(R)\right]_{\mathrm{pole}}=} & {\left[R^{*}(t)\right]^{-1}\left(1-\frac{\epsilon}{7} \frac{d \operatorname{Re} H(-1)}{d z}\right) } \\
& \times \exp \left[-\left(\frac{R}{R^{*}(t)}\right)\left(1+\frac{\epsilon}{7} \operatorname{Re} H(-1)\right)\right]
\end{aligned}
$$

and, except when $R=0$ (for this case see the Appendix), $\left[P_{c}(R)\right]_{\mathrm{cut}}$ is 


$$
\begin{aligned}
{\left[P_{c}(R)\right]_{\mathrm{cut}}=} & -\frac{\epsilon}{7}\left[R^{*}(t)\right]^{-1} \int_{0}^{\infty} \exp \left[-R(1+x) / R^{*}(t)\right] \frac{1}{1+x} d x \\
& \left.+\frac{\epsilon}{42}\left[R^{*}(t)\right]^{-1} \int_{0}^{\infty} \exp \left[-R(4+x) / R^{*}(t)\right] \frac{x}{(x+3)^{2}} \mid \frac{x}{x+4}\right]^{1 / 2} d x,
\end{aligned}
$$

where, as can be found from Eq. (26),

$$
\operatorname{Re} H(-1)=\frac{25}{18}-\pi \sqrt{3} / 6
$$

and

$$
d \operatorname{Re} H /\left.d y\right|_{-1}=-\frac{5}{9}-\pi \sqrt{3} / 6 .
$$

To verify Eq. (36) we used it to calculate the moments,

$$
\int_{0}^{\infty} P_{c}(R) d R=1, \quad \int_{0}^{\infty} P_{c}(R) R^{q} d R=m_{q}=\chi^{(q)} / \chi_{p} .
$$

We used these $m_{q}$ 's to explicitly construct the quantities $\rho_{q} \equiv m_{q} /\left(m_{1}\right)^{q}$, for $q \leq 4$. These values of $\rho_{q}$ were checked by comparing them to those calculated from the $\delta_{q}$ 's of Eq. (28), as follows. Combining Eqs. (10), (12), and (13) we have that

$$
\begin{aligned}
\chi_{R}(\lambda) & =\sum_{r}\left(-\frac{1}{2} \lambda^{2}\right)^{r} \frac{1}{r !} \chi^{(r)} \\
& =\chi^{(0)} \sum_{r} \rho_{r}\left(\chi^{(1)} / \chi^{(0)}\right)^{r}\left(-\frac{1}{2} \lambda^{2}\right)^{r} \frac{1}{r !} .
\end{aligned}
$$

Alternatively, Eq. (27) with $\Gamma_{k}$ as given just after that equation is

$$
\chi_{R}(\lambda)=\left(A t^{\gamma} \sum_{p} \delta_{p} a^{p} \lambda^{2 p} t^{-p \phi}\right)^{-1}
$$

Comparing these two equations, we see that $\chi^{(0)}$ $=\left[A t^{\gamma}\right]^{-1}, \chi^{(1)} / \chi^{(0)}=2 a t^{-\phi}$, and

$$
\begin{aligned}
& \rho_{2}=2\left(1-\delta_{2}\right), \\
& \rho_{3}=6\left(1-2 \delta_{2}+\delta_{3}\right),
\end{aligned}
$$

and so forth.

\section{Phase diagram for RRN and RXY models}

Several comments on the preceding results are in order. For $p$ near $p_{c}$, i.e., for large $y$ we can analyze Eq. (25) to see whether the function $F(y) y^{-\gamma / \phi}$ is an analytic function of $\left(p-p_{c}\right) / w^{1 / \phi}=y^{1 / \phi}$. One finds that this is not the case, so that $\chi_{R}(t, \lambda)$ is nonanalytic for $p=p_{c}$ (i.e., for $t=0$ ) even for nonzero $\lambda$. This result is easily understood in the following way. Normally one has a propagator of the form

$$
\hat{G}^{-1}(t, q)=t+q^{2},
$$

for, say, an Ising model, where $t=\left(T-T_{c}\right)$. A literal interpretation of this result would indicate that $\hat{G}(t, q)$ for nonzero $q$ becomes singular at a $q$-dependent critical value of $t: t_{c}(q)=t_{c}(0)-q^{2}$. This is wrong, of course, and the reason it is wrong is that $\hat{G}^{-1}(q)$ for nonzero $q$ is coupled by fluctuations to smaller $q$ 's, and in particular, to $q=0$. Here, we have the same effect as a function of $\lambda$. However, to be more precise, we should say that the phase transition occurs when $\hat{G}\left(t, q=0, \lambda_{\min }\right)$ becomes singular. (For more details see Ref. 15.) However, for the resistor network $\lambda_{\min }=\pi / V_{\max }$, where $V_{\max }$ is the largest allowed value of the voltage. To recover the resistor network one must take the limit $V_{\max } \rightarrow \infty$, so that the phase transition for the RRN occurs at $p_{c}$ for all values of $w \equiv w_{1} \lambda^{2}$. A phase diagram for $\chi_{R}(\lambda)$ in which $\lambda$ is a variable is implausible. This argument is illuminating in that it shows why and how the RXY model can have a different phase diagram from the RRN. In particular, for the RXY model $V$ is replaced by a spin angle and we have that $V_{\max }=\pi$, so that $\lambda_{\min }=1$. Thus the critical value of $\left(p-p_{c}\right)$ for the RXY model is determined by when $\chi_{R}\left(t, \lambda_{\min }\right)$, with $\lambda_{\min }=1$, becomes singular. Within mean-field theory and setting $w=T / J$, this happens when

$$
p=p_{c}+T / J .
$$

The details of how this phase boundary is modified by fluctuations for $d=6-\epsilon$ will be given elsewhere. ${ }^{15}$

\section{SERIES RESULTS}

In order to check the predictions of the $\epsilon$ expansion we have constructed series for several moments of the resistance, $\chi^{(k)}$, which we assume to diverge as

$$
\chi^{(k)}=\left\langle\sum_{\mathbf{x}}[R(0, \mathbf{x})]^{k}\right\rangle_{\mathrm{av}} \sim A_{k} t^{-\gamma_{k}},
$$

where ${ }^{7} \gamma_{k}=\gamma+k \phi$, and recall that $\chi^{(0)}$ denotes the percolation susceptibility. The series for $\chi^{(0)}$ was published up to 11 th order by Adler et al. ${ }^{20}$ The series for $\chi^{(1)}$ up to 10th order is given by Fisch and Harris ${ }^{7}$ and one further term is given by Harris et al. ${ }^{21}$ In Table I we give the coefficients needed to construct the series for $\chi^{(2)}$ in arbitrary dimension up to 11 th order in $p$. In order to get estimates for the amplitude ratio we used a newly proposed method $^{22}$ in which one calculates the universal quantity

$$
S_{i j / k l} \equiv \frac{A_{i} A_{j}}{A_{k} A_{l}} \frac{\Gamma\left(\gamma_{k}\right) \Gamma\left(\gamma_{l}\right)}{\Gamma\left(\gamma_{i}\right) \Gamma\left(\gamma_{j}\right)},
$$

where $\Gamma(x)$ is the factorial function. In this method one multiplies $(M)$ and divides $(D)$ the series term by term as follows to form the quantity

$$
\left[\chi^{(i)} \boldsymbol{M} \chi^{(j)}\right] \boldsymbol{D}\left[\chi^{(k)} \boldsymbol{M} \chi^{(l)}\right] \equiv \boldsymbol{\Phi}_{i j / k l} \text {. }
$$

The result of this operation is to obtain a quantity $\Phi$ which diverges with a known exponent at a known concentration:

$$
\Phi_{i j / k l} \sim S_{i j / k l}(1-p)^{-1}
$$


TABLE I. Series coefficients for $\chi_{2}$ in $d$ dimensions: $\chi^{(2)}=\sum_{k l} a(k, l) p^{k} d^{l}$. To save space we introduce the notation [ $n$ ], which indicates that the value of $a(k, l)$ is $10^{n}$ times the value listed in the table.

\begin{tabular}{rrrrrrrrr}
\hline \hline$k$ & $l$ & $a(k, l)$ & $k$ & $l$ & $a(k, l)$ & $k$ & $l$ \\
\hline 1 & 1 & $0.1000000000[01]$ & 2 & 1 & $-0.4000000000[01]$ & 2 & 2 & $0.8000000000[01]$ \\
3 & 1 & $0.9000000000[01]$ & 3 & 2 & $-0.3600000000[02]$ & 3 & 3 & $0.3600000000[02]$ \\
4 & 1 & $0.4187500000[02]$ & 4 & 2 & $0.3812500000[02]$ & 4 & 3 & $-0.1920000000[03]$ \\
4 & 4 & $0.1280000000[03]$ & 5 & 1 & $-0.3165000000[03]$ & 5 & 2 & $0.5330000000[03]$ \\
5 & 3 & $0.2085000000[03]$ & 5 & 4 & $-0.8000000000[03]$ & 5 & 5 & $0.4000000000[03]$ \\
6 & 1 & $-0.1341472222[04]$ & 6 & 2 & $0.3944166667[03]$ & 6 & 3 & $0.1605555556[04]$ \\
6 & 4 & $0.1105500000[04]$ & 6 & 5 & $-0.2880000000[04]$ & 6 & 6 & $0.1152000000[04]$ \\
7 & 1 & $0.1490232056[05]$ & 7 & 2 & $-0.3004121944[05]$ & 7 & 3 & $0.1188134333[05]$ \\
7 & 4 & $0.4470555556[04]$ & 7 & 5 & $0.5108000000[04]$ & 7 & 6 & $-0.9408000000[04]$ \\
7 & 7 & $0.3136000000[04]$ & 8 & 1 & $0.1264635833[06]$ & 8 & 2 & $-0.1693400356[06]$ \\
8 & 3 & $0.9862017778[04]$ & 8 & 4 & $0.2159976778[05]$ & 8 & 5 & $0.1118066667[05]$ \\
8 & 6 & $0.2077800000[05]$ & 8 & 7 & $-0.2867200000[05]$ & 8 & 8 & $0.8192000000[04]$ \\
9 & 1 & $-0.1196556276[07]$ & 9 & 2 & $0.2968259558[07]$ & 9 & 3 & $-0.2346641608[07]$ \\
9 & 4 & $0.5118622285[06]$ & 9 & 5 & $0.2571532000[05]$ & 9 & 6 & $0.2320977778[05]$ \\
9 & 7 & $0.7644000000[05]$ & 9 & 8 & $-0.8294400000[05]$ & 9 & 9 & $0.2073600000[05]$ \\
10 & 1 & $-0.1521443954[08]$ & 10 & 2 & $0.2797756385[08]$ & 10 & 3 & $-0.1279170017[08]$ \\
10 & 4 & $-0.1258880240[07]$ & 10 & 5 & $0.1194181531[07]$ & 10 & 6 & $-0.1742454667[05]$ \\
10 & 7 & $0.2984711111[05]$ & 10 & 8 & $0.2601520000[06]$ & 10 & 9 & $-0.2304000000[06]$ \\
10 & 10 & $0.5120000000[05]$ & 11 & 1 & $0.1201248606[09]$ & 11 & 2 & $-0.3486761447[09]$ \\
11 & 3 & $0.3634394156[09]$ & 11 & 4 & $-0.1548229566[09]$ & 11 & 5 & $0.1713212635[08]$ \\
11 & 6 & $0.2762746081[07]$ & 11 & 7 & $-0.2529129778[06]$ & 11 & 8 & $-0.4429333333[05]$ \\
11 & 9 & $0.8328960000[06]$ & 11 & 10 & $-0.6195200000[06]$ & 11 & 11 & $0.1239040000[06]$ \\
\hline \hline
\end{tabular}

The advantage of this method is that the estimates for $S$ do not rely on estimates of the critical point, $p_{c}$, or of any of the critical exponents. We list in Table II our estimates for several such amplitude ratios. In order to compare to the $\epsilon$ expansion we list the $\epsilon$-expansion results for these ratios. One can see that although the values of the ratios $R_{i j / k l} \equiv A_{i} A_{j} / A_{k} A_{l}$ are nontrivial, the quantities $S_{i j / k l}$ are very close to unity in all dimensions. Also, since the coefficient of $\epsilon$ in $S$ is so small, the $\epsilon^{2}$ term might be the larger correction to unity for several ratios.
In view of this, one might be tempted to get an estimate for the resistance distribution for all dimensions by equating $S_{i j / k l}$ to unity for all dimensions and all subscripts. However, although this may be a good numerical approximation for any given $S$, it leads to

$$
\rho_{q}=\Gamma(\gamma+q \phi) \Gamma(\gamma)^{q-1} / \Gamma(\gamma+\phi)^{q} \text {. }
$$

Inserting this ansatz into the right-hand side of Eq. (38) yields

TABLE II. Estimates of the amplitude ratios. I: Series, II: $\epsilon$ expansion (Ref. 13) [using the $\epsilon$ expansion for the exponents (Refs. 9,10 , and 13)].

\begin{tabular}{|c|c|c|c|c|c|c|}
\hline & $d$ & 6 & 5 & 4 & 3 & 2 \\
\hline \multicolumn{7}{|c|}{$S_{02 / 11}$} \\
\hline I & & 1 & $1.00 \pm 0.001$ & $1.005 \pm 0.001$ & $1.009 \pm 0.001$ & $1.006 \pm 0.02$ \\
\hline II: & $1+\frac{\epsilon}{420}$ & 1 & 1.002 & 1.005 & 1.007 & 1.010 \\
\hline \multicolumn{7}{|c|}{$S_{03 / 12}$} \\
\hline I & & 1 & $1.005 \pm 0.001$ & $1.002 \pm 0.001$ & $1.001 \pm 0.001$ & $0.986 \pm 0.004$ \\
\hline & $1+\frac{\epsilon}{392}$ & 1 & 1.003 & 1.005 & 1.008 & 1.010 \\
\hline \multicolumn{7}{|c|}{$S_{13 / 22}$} \\
\hline I & & 1 & $0.999 \pm 0.001$ & $0.997 \pm 0.001$ & $0.992 \pm 0.002$ & $0.98 \pm 0.01$ \\
\hline II: & $1+\frac{\epsilon}{5880}$ & 1 & 1.000 & 1.000 & 1.001 & 1.001 \\
\hline \multicolumn{7}{|c|}{$S_{04 / 22}$} \\
\hline I & & 1 & $0.998 \pm 0.001$ & $0.993 \pm 0.002$ & $0.98 \pm 0.01$ & $0.94 \pm 0.02$ \\
\hline II: & $1+\frac{103 \epsilon}{52920}$ & 1 & 1.001 & 1.004 & 1.006 & 1.008 \\
\hline I & & \multicolumn{5}{|c|}{$S_{14 / 23}$} \\
\hline II: & $1-\frac{32 \epsilon}{52920}$ & 1 & 0.999 & 0.999 & 0.998 & 0.998 \\
\hline
\end{tabular}




$$
\chi_{R}(t, \lambda)=\chi^{(0)} \sum_{n=0}^{\infty} \frac{\Gamma(\gamma+n \phi)}{\Gamma(\gamma) n !}(-y)^{n},
$$

where

$$
y=\frac{1}{2} \lambda^{2} \chi^{(1)} \Gamma(\gamma) /\left[\Gamma(\gamma+\phi) \chi^{(0)}\right] .
$$

However, this series does not converge for $d<6$, where $\phi$ is larger than unity. The small deviations of $S_{i j / k l}$ from unity are thus crucial for obtaining the full distribution function $P_{c}(R)$.

\section{CONCLUSIONS}

This paper is the first of a series of three papers which aim to study in detail (a) the probability distribution $P_{c}(R, \mathrm{x})$ for the resistance $R$ between two terminals at separation $\mathbf{x}$, known to be in the same cluster and (b) the phase boundary between the ordered phase and the disordered phase. In this paper we have concentrated on the average of $P_{c}(R, \mathbf{x})$ over $\mathbf{x}$. This was done using (a) field theory and (b) series expansions. The $\epsilon$ expansion for the generalized resistive susceptibility, $\chi_{R}(t, \lambda)$, defined in Eq. (13), is given in Eqs. (24)-(26). The resulting scaling function is consistent with the $\epsilon$ expansion for the amplitude ratios of the resistive susceptibilities given by Harris and Lubensky. ${ }^{13}$ The amplitude ratios were also estimated from series expansions, using a newly developed method, ${ }^{22}$ to yield results that agree within a few percent with the $\epsilon$ expansion (See Table II). The resulting probability distribution, obtained by inverse Laplace transformation of $\chi_{R}(t, \lambda)$, is given in Eq. (36). We also discussed why one should expect a different phase diagram for the RRN than for the RXY model.

\section{ACKNOWLEDGMENTS}

A.B.H. would like to thank the School of Physics and Astronomy of Tel Aviv University for its hospitality. We acknowledge support from the U.S.-Israel Binational Science Foundation, the Israel Academy of Sciences and Humanities, and the National Science Foundation under Grant No. 85-20272.

\section{APPENDIX: EVALUATION OF $\boldsymbol{\Lambda}(\boldsymbol{x})$}

In this appendix we evaluate $\Lambda(x)$ given by

$$
\Lambda(x)=\frac{1}{2 \pi i} \int_{a-i \infty}^{a+i \infty} \frac{e^{z x}}{1+z+\frac{\epsilon}{7} H(z)} d z,
$$

where $H(z)$ is given by

$$
\begin{aligned}
H(z)= & \frac{(1+z)^{2}}{z} \ln (1+z)+\frac{6-19 z}{18} \\
& -\frac{(z+4)}{6}\left(\frac{z+4}{z}\right)^{1 / 2} \ln \left(\frac{\sqrt{z+4}+\sqrt{z}}{\sqrt{z+4}-\sqrt{z}}\right) .
\end{aligned}
$$

The logarithms and square roots are defined to be real positive when their arguments are real positive. The function $H(z)$ is defined so as to be analytic except for a branch cut running along the negative real $z$ axis from $z=-1$ to $-\infty$. For small $\epsilon$ one sees that poles in the integrand of Eq. (A1) can only occur (a) near $z=-1$, or (b) at large $|z|$ where $(\epsilon / 7) H(z)$ may become of order unity. Case (b) can be ruled out because for large $|z|$ we have

$1+z+(\epsilon / 7) H(z) \sim z\left[1+\frac{\epsilon}{7}\left[\frac{5}{6} \ln z-\frac{19}{18}\right)\right] \neq 0$,

and case (a) is ruled out because $\operatorname{Re} H(-1)>0$ and $\operatorname{Im} H(-1) \sim(z+1)^{2}$ for $z$ near -1 .

We may therefore deform the path of integration into a contour $C$ surrounding the branch cut. For the moment, we assume that $x$ is not near zero (as we shall see, this means that $x / \epsilon \gg>1)$. Then the factor $e^{2 x}$ makes the integral strongly convergent and allows manipulations of the denominator, which we write as

$$
\begin{aligned}
1+z & +\frac{\epsilon}{7} H(-1)+\frac{\epsilon}{7} H^{\prime}(-1)(z+1)+\frac{\epsilon}{7} \delta H(z) \\
& \approx\left(1+\frac{\epsilon}{7} H^{\prime}(-1)\right]\left[z+1+\frac{\epsilon}{7} H(-1)\right)+\frac{\epsilon}{7} \delta H(z) \\
& \equiv A\left(z+z_{0}\right)+\frac{\epsilon}{7} \delta H(z),
\end{aligned}
$$

where $z_{0} \approx 1, A \approx 1$, and $\delta H(z)$ is of order $(z+1)^{2}$ for $z$ near 1. In fact, we treat $\delta H(z)$ as being of order $\left(z+z_{0}\right)^{2}$, since $z_{0}=1$ to order $\epsilon$. Thus to order $\epsilon$ we have

$$
\begin{aligned}
\Lambda(x) & =\frac{1}{2 \pi i} \int_{C} e^{z x} d z\left(\frac{1}{A\left(z+z_{0}\right)}-\frac{\epsilon}{7} \frac{\delta H(z)}{A^{2}\left(z+z_{0}\right)^{2}}\right) \\
& =\frac{1}{A} e^{-x z_{0}}+\frac{\epsilon}{7} \int_{1}^{\infty} \frac{e^{-z x}}{(z+1)^{2}}\left(\frac{H\left(-z+i 0^{+}\right)-H\left(-z-i 0^{+}\right)}{2 \pi i}\right) d z,
\end{aligned}
$$

which leads to Eq. (36).

For small $x$ Eq. (36) gives

$$
\Lambda(x) \sim \Lambda_{0}+c \epsilon \ln x,
$$

where $\Lambda_{0}$ is $\Lambda(0)$ for $\epsilon=0$ and $c$ is a constant of order unity. Such a result is absurd because physically $\Lambda(x) \geq 0$, whereas Eq. (A6) gives $\Lambda(0)=-\infty$. Of course, for $x=0$ the expansion in Eq. (A5a) is qualitatively incorrect at sufficiently large $|z|$. To obtain the correct result for $x=0$ we simply analyze correctly the contribution to $\Lambda(x)$ from large $|z|$ when the contour is deformed 
to surround the branch cut. By large $|z|$ we mean $|z|$ such that $(\epsilon / 7) H(z)$ is comparable to $1+z$. This happens for $|(5 \epsilon / 42) z \ln z| \sim|z|$, or $|z| \sim \exp [1 /(c \epsilon)]$, where $c=\frac{5}{42}$, as in Eq. (A6). Thus we estimate that

$$
\begin{aligned}
\Lambda(0) & =\Lambda_{0}+\frac{1}{2 \pi i} \int_{e^{1 /(c \epsilon)}}^{\infty} d z\left\{[z+c \epsilon z(\ln z+\pi i)]^{-1}-\text { c.c. }\right\} \\
& \approx \Lambda_{0}-\frac{1}{2 \pi i} \int_{e^{1 /(c \epsilon)}}^{\infty} \frac{d z}{z c \epsilon} \frac{2 \pi i}{(\ln z)^{2}}
\end{aligned}
$$

$$
\approx \Lambda_{0}-\frac{1}{c \epsilon} \int_{1 /(c \epsilon)}^{\infty} \frac{d u}{u^{2}}=\Lambda_{0}-1
$$

Presumably there is a crossover such that

$$
\begin{aligned}
\Lambda(x) & =\Lambda_{0}-\text { const, } \quad x<e^{-(1 / c \epsilon)}, \\
& =\Lambda_{0}+c \epsilon \ln x, \quad e^{-(1 / c \epsilon)}<<x<<1,
\end{aligned}
$$

but we do not pursue this analysis further here.
*Permanent address: Department of Physics, University of Pennsylvania, Philadelphia, PA 19104.

1J. W. Essam, Rep. Progr. Phys. 43, 843 (1980).

${ }^{2}$ D. Stauffer, Introduction to Percolation Theory (Taylor and Francis, London, 1985).

${ }^{3}$ A. Aharony, in Directions in Condensed Matter Theory, edited by G. Grinstein and G. Mazenko (World Scientific, Singapore, 1986), p. 1.

${ }^{4}$ B. J. Last and D. J. Thouless, Phys. Rev. Lett. 27, 1719 (1971).

${ }^{5}$ A. S. Skal and B. I. Shklovskii, Fiz. Tekh. Poluprovodn. 8, 1582 (1974) [Sov. Phys. - Semicond. 8, 1029 (1975)].

${ }^{6}$ P. G. de Gennes, J. Phys. (Paris) Lett. 37, L1 (1976).

${ }^{7}$ A. B. Harris and R. Fisch, Phys. Rev. Lett. 38, 796 (1977); R. Fisch and A. B. Harris, Phys. Rev. B 18, 416 (1978).

${ }^{8}$ J. G. Zabolitzky, Phys. Rev. B 30, 4077 (1984); C. J. Lobb and D. J. Frank, ibid. 30, 4090 (1984); H. J. Herrmann, B. Derrida, and J. Vannimenus, ibid. 30, 4080 (1984); D. C. Hong, S. Havlin, H. J. Herrmann, and H. E. Stanley, ibid. 30, 4083 (1984).

${ }^{9}$ A. B. Harris, S. Kim, and T. C. Lubensky, Phys. Rev. Lett. 53, 743 (1984); 54, 1088(E) (1985).
${ }^{10}$ A. B. Harris and T. C. Lubensky, J. Phys. A 17, L609 (1984).

${ }^{11}$ R. B. Stinchcombe and B. P. Watson, J. Phys. C 9, 3221 (1976).

${ }^{12}$ R. Rammal, M. A. Lemieux, and A.-M. S. Tremblay, Phys. Rev. Lett. 54, 1087(C) (1984).

${ }^{13}$ A. B. Harris and T. C. Lubensky, Phys. Rev. B 35, 6964 (1987).

${ }^{14}$ A. B. Harris and A. Aharony (unpublished).

${ }^{15}$ A. B. Harris and A. Aharony, Phys. Rev. B 40, 7230 (1989).

${ }^{16}$ A. B. Harris, T. C. Lubensky, W. K. Holcomb, and C. Dasgupta, Phys. Rev. Lett. 35, 327 (1975); 35, 1397(E) (1975).

${ }^{17}$ A. Aharony, Phys. Rev. B 22, 400 (1980), and references therein.

${ }^{18}$ M. J. Stephen, Phys. Rev. B 17, 4444 (1978).

${ }^{19}$ K. G. Wilson, Phys. Rev. Lett. 28, 548 (1972).

${ }^{20}$ J. Adler, A. Aharony, Y. Meir, and A. B. Harris, J. Phys. A 19, 3631 (1986).

${ }^{21}$ A. B. Harris, Y. Meir, and A. Aharony, Phys. Rev. B 36, 8752 (1987).

${ }^{22}$ Y. Meir, J. Phys. A 20, L349 (1987). 